AROUEOLOGÍA Y SOCIEDAD

№ 26, 2013: 249-264

ISSN: 0254-8062

RECIBIDO: 30 / MAR. / 2013 ACEPTADO: 5 / MAY. / 2013

\title{
EL USHNU DE HUÁNUCO PAMPA: UNA PLATAFORMA CEREMONIAL INCA COMO LUGAR DE LIBACIONESY EJE DE PLANIFICACIÓN DE UN CENTRO ADMINISTRATIVO-RELIGIOSO EN LOS ANDES CENTRALES
}

\author{
JosÉ LUIS PINO MATOS* \\ Magister en Arqueología. Miembro del icomos y del Programa de Estudios Andinos - PCUP \\ josepinomatos@gmail.com
}

\section{RESUMEN}

Se propone, en este artículo, que los componentes arquitectónicos de la plataforma ushnu de Huánuco Pampa, están vinculados espacialmente con el paisaje circundante y que los rituales de libación fueron la actividad pública más importante, razón por la cual esta plataforma, además de construirse al centro de todo el asentamiento, sirvió como principio de planificación del centro administrativo-religioso inca de Huánuco Pampa.

Palabras Clave: sociedad inca, plataforma ushnu, planificación de asentamientos, libaciones rituales.

\section{Abstract}

The proposal in this article is, that the architectural components of the ushnu platform of Huánuco Pampa are spatially associated with the surrounding landscape and that libation rituals were the most important public activity, of this mode, this platform, was built at the center of this settlement and served as planning principle of the Inca administrative-religious center of Huánuco Pampa.

KEYworDs: Inca society, ushnu platform, settlement planning, ritual libations.

\section{ANTECEDENTES DE INVESTIGACIÓN SOBRE EL USHNU}

El ushnu es conocido como un lugar ceremonial donde principalmente se ofrecía chicha ${ }^{1}$, esta interpretación se basa en la revisión de información etnohistórica (Pino 2010a) y en la información re-

1 La chicha es una bebida fermentada de maíz, la palabra chicha, sin embargo, es una denominación centroamericana, pero que en los Andes a esta bebida se le conoce como aswa o aqha en lengua quechua. 
ferida por algunos estudios precedentes. Sin duda, uno de los pioneros en plantear una temática de investigación sobre el ushnu, fue el antropólogo holandés Tom Zuidema. Sin embargo, la utilización de la palabra ushnu en los estudios andinos no empieza con Zuidema, sino probablemente se remonta al año 1965 durante la ejecución del Proyecto de la Vida Provincial Incaica del Institute of Andean Research dirigido por John Murra, proyecto en el que participó el antropólogo cusqueño Dr. Manuel Chávez Ballón, quien sugiere que a la plataforma ceremonial inca se denomine Usnu o de acuerdo al quechua o runa simi local de la provincia de Dos de Mayo (Huánuco), se refiera con la palabra ushnu a la estructura central del sitio inca de Huánuco Pampa (Shea 1966: 108).

Posteriormente los arqueólogos Donald Thompson y Craig Morris utilizan dicho término para denominar las construcciones ubicadas en el centro de las plazas de los asentamientos incas (Morris y Thompson 1970); seguidamente otros arqueólogos generalizaron con este nombre a plataformas y altares que se encontraron en las plazas de los principales sitios incas del Chinchaysuyu. En este estado del desarrollo del estudio del ushnu, es donde Zuidema publica sus propuestas sobre el mismo en 1980. Y a partir de ahí se asociaría en el mayor de los casos a ushnu como un lugar compuesto de plataformas en los principales asentamientos incas, y casi siempre esta palabra asociada a la sociedad inca.

A la fecha se han realizado varios estudios sobre el ushnu inca, sobre todo por arqueólogos Matos (1986), Hyslop (1990), Meddens (1997), Meddens et al. (2008), quienes al igual que Tom Zuidema (1980) y John Rowe (1990) utilizan las fuentes etnohistóricas para las interpretaciones de su función y significado. De igual forma Graziano Gazparini y Louise Margolies en su estudio sobre arquitectura inca tratan de definir entre la edificaciones inca ¿Qué es un ushnu?, y dejan entrever que se trataría de una especie de trono y altar (Gazparini y Margolies 1977: 274-288).

Como se indicó, varios estudios describen algunas características físicas de los ushnus en el Tawanatinsuyu, estas descripciones están presentes además de las anteriormente citadas en los estudios de Rodolfo Raffino y colaboradores en la zona sur del imperio inca (Raffino et al. 1997), en la zona del Cuntisuyu (Ziołkowski 2008); así como en la zona central de los andes denominada como la zona central del Chinchaysuyu, en Pumpu y Chakamarca por Ramiro Matos (1986, 1994, 1995) y Matos, Arellano y Brown (1996); en las alturas de Ayacucho por Frank Meddens y colaboradores (2008); en los estudios de sitios de la costa central reportados por Makowski y su equipo (Makowski et al. 2008; Makowski 2011) y otros en el valle del Rímac (Villacorta 2005); como también en Huánuco Pampa por Shea (1966), Morris y Thompson $(1970,1985)$ posteriormente de igual forma por José Pino (2004a, 2004 b y 2005). (Fig. 1).

\section{Referencias previas sobre el ushnu de HuÁnuco PAMPA}

Entre las referencias previas a considerar sobre Huánuco Pampa, se encuentra la del arquitecto Emilio Harth-Terré, quien visitó el sitio inca denominándolo, aún para aquel entonces, como Huánuco Viejo y refiere a la plataforma central como «Castillo» (Harth-Terré 1964), en base a la referencia de los viajeros, describiéndolo de la siguiente manera:

«Es una construcción rectangular de 38 metros por 63 m. que se sitúa en el medio mismo de la plaza. La altura de sus muros no sobrepasa los $3.7 \mathrm{~m}$. a $4 \mathrm{~m}$. y se elevan sobre una plataforma de $1 \mathrm{~m}$. de ancho y $0.80 \mathrm{~m}$. de altura sobre el plano de la plaza, con muros de cantería, que aunque rústicos se aprecia en ellos la ingeniosa técnica del inca» (Harth-Terré 1964: 7).

Sobre el eje del «Castillo» y su asociación con la ruta que recorre el conjunto de portadas, indica que: «Por todo lo dicho, es indudable que no se trata de un edificio castrense. El nombre de "castillo" es inapropiado a todas luces. Seguiremos sin embargo empleándolo por la costumbre impuesta. A mi criterio era edificio para ceremonias y rituales. Más si consideramos que el eje longitudinal del edificio corresponde a un eje ideal, el de las cuatro puertas del Incahuasi. Esto acaba de vincularlo a todo el complejo urbano» (Harth-Terré 1964: 7). 


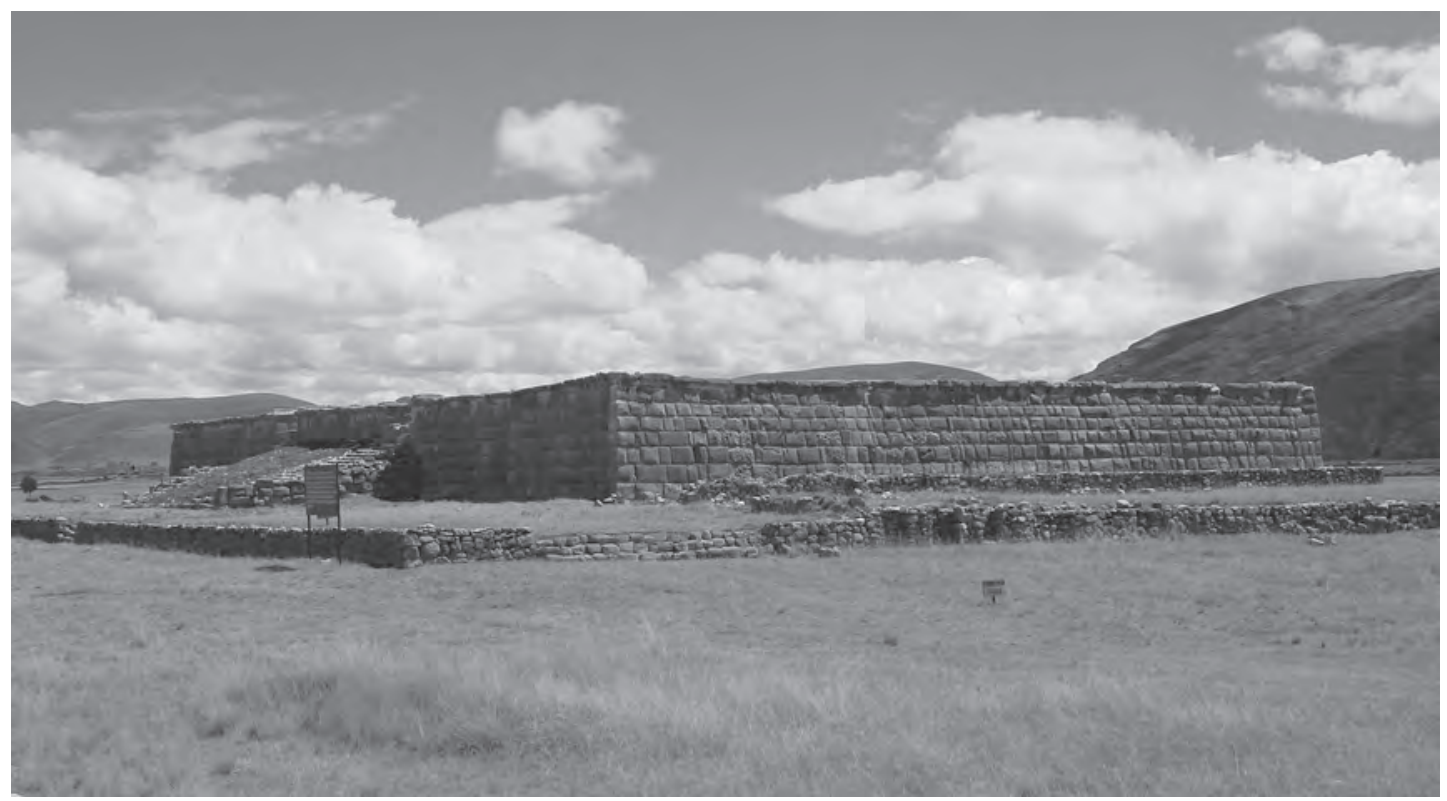

Figura 1. Plataforma Ushnu de Huánuco Pampa.

Posteriormente otro investigador norteamericano, Dan Shea, refiere que la utilización de la palabra ushnu en los estudios andinos no empieza con Zuidema, sino con el antropólogo cusqueño Dr. Chávez Ballón que sugiere se denomine usnu, o de acuerdo al runa simi local de la provincia de Dos de Mayo (Huánuco) con la palabra ushnu, a la estructura central del sitio inca de Huánuco Pampa (Shea 1966: 108), en el año 1965 durante la ejecución del Proyecto de la Vida Provincial Incaica del Institute of Andean Research dirigido por John Murra.

Shea describe la plaza y el edificio central dentro de ella $(1966,1968)$. La plaza que es considerada enorme, mide cerca de 350 x $550 \mathrm{~m}$, y en su centro se asienta el ushnu, una plataforma sólida de destacada albañilería inca imperial. El ushnu, el cual mide aproximadamente 32 x $48 \mathrm{~m}$ en la base y aproximadamente 3,5 $\mathrm{m}$ de alto, parece estar dispuesto sobre dos terrazas bajas, la más baja exterior, aproximadamente de $12 \mathrm{~m}$ de ancho y en el interior otra superior de 1,5 $\mathrm{m}$ de ancho. Dos pequeños edificios sobre el lado este de la terraza baja podrían haber funcionado en conexión con el ushnu en las ceremonias llevadas a cabo en esta plataforma (Shea 1968: 19-21).

Los estudios de Shea indican que para acceder a la parte superior del ushnu, se realiza por una escalera sobre el lado sur, encontrada en muy mal estado, pero se estima que debió haber tenido 32 pasos (Shea 1968: 17). La parte superior está encerrada por un muro bifacial de más de un metro de grosor y 1,5 $\mathrm{m}$ de alto. Una pequeña proyección exterior de la parte superior del muro produce una cornisa alrededor de la parte superior del ushnu. Esta plataforma posee dos entradas, cada uno de casi $3 \mathrm{~m}$ de ancho, proporcionan el acceso hacia la parte superior de la estructura desde las escaleras. Las entradas se encuentran flanqueadas por unas piezas escultóricas de piedra, pequeñas y pobremente ejecutadas, probablemente representando un par de pumas que se dan la espalda. El muro alrededor de la parte superior del ushnu también contuvo 10 nichos, 6 de los cuales son aun claramente visibles. Estos nichos no tuvieron partes superiores y pudieron fácilmente haber servido como asientos (Shea 1966). (Fig. 2).

De igual manera, casi de forma paralela a las investigaciones de Dan Shea, en Huánuco Pampa, por esos años se llevaron a cabo las investigaciones de Craig Morris y Donald Thompson. Estos inves- 
tigadores indican que todo el sitio fue diseñado alrededor de una enorme plaza abierta de 550 x 350 m. Una fina albañilería de piedra fue utilizada para la construcción la plataforma al centro de la plaza. Plataforma que posiblemente fue asociada con actividades ceremoniales. Morris y Thompson proponen que posiblemente el Inca o algún representante pudieron haberse sentado en la plataforma para hacer observaciones astronómicas y oficiar en las ceremonias en las actividades del Estado (Morris y Thompson 1985: 58-59). De igual manera estos autores refieren que el ushnu en Huánuco Pampa se asienta sobre dos plataformas bajas, su altura por encima de la plataforma más alta es aproximadamente 3,5 m. Las escalinatas ascienden desde el lado sur, y está rodeada por un parapeto y se puede ingresar a través de dos entradas. Las entradas en la cima del ushnu están flanqueadas sobre cada lado por figuras de animales duales esculpidos en alto relieve. Los animales, probablemente son pumas y están dispuestos así que una de cada par mira el interior de la estructura, las otras el exterior. Un animal similar, visto de perfil, adorna una piedra en el lado oeste de las estructura cerca de la esquina noroeste (Morris y Thompson 1985: 59).

El ushnu o plataforma principal posee la forma de un rectángulo perfecto, con dimensiones de 32 x $48 \mathrm{~m}$ en la base. Sólo los lados expuestos de las piedras grandes de las cuales está construida, estan alisadas. Los lados no expuestos y posteriores fueron dejados sin pulimentar y fijados en un relleno de piedras y tierra. Así, las piedras pulidas crean elegantemente una fachada de bloques rectangulares. Las caras de las plataformas bajas y los escalones no están trabajados. La plataforma más baja aparentemente tuvo la función de crear una superficie nivelada sobre la cual se ubica la estructura principal. La mitad este de la plaza se inclina un poco, y la plataforma más baja corrige esto. La pequeña plataforma superior fue construida después de la estructura principal de piedra pulida. Ambas plataformas aumentan la escala y el impacto visual de la estructura, eliminando la impresión de una simple torre construida en un gran espacio abierto. Morris y Thompson también mencionan la presencia de figuras de animales tallados en otras partes del sitio arqueológico bastante idénticas a las que están sobre el muro oeste del ushnu. Las portadas del lado este del asentamiento están tan alineadas que se puede ver a través de ellas hacia un punto del muro este del ushnu. La orientación de esta línea es aproxima-

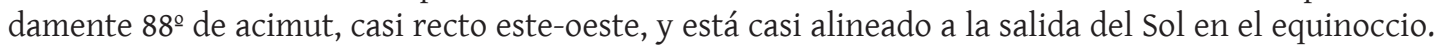
Parece claro que las portadas conectan la plaza central, el ushnu, los grandes recintos con las dos plazas secundarias y sus edificios públicos, y el conjunto de fina arquitectura y fuentes de agua dentro de una sola unidad. Excavaciones de prueba en varios lugares revelan un canal siguiendo esencialmente la misma línea exacta de dirección las portadas (Morris y Thompson 1985: 59-60).

Craig Morris manifiesta que los ushnu se trataban de plataformas rectangulares especiales con una función eminentemente ceremonial y manifiesta que:

«Las excavaciones dentro y alrededor de algunas de estas plataformas revelaron vasijas de cerámica y restos de alimentos quemados. La naturaleza y el contexto de estos materiales sugieren que las plataformas fueron aparentemente usadas como altares. Las ofrendas se depositaban en los altares o a los pies de estos. Otro rasgo que a veces sirve para identificar las estructuras religiosas es un hoyo excavado en el piso y revestido de piedras. Llenos de guijarros redondos de río, estos hoyos podrían haber sido usados para las ofrendas de chicha $u$ otro líquido que se vertía sobre las piedras y otros aspectos del paisaje natural fueron frecuentemente considerados sagrados por los incas, su incorporación a la arquitectura es comprensible, y a veces la evidencia arqueológica de ofrendas se encuentra asociada con esos aspectos naturales» (Morris 1999: 27).

Asimismo, Morris y Thompson plantean que Huánuco Pampa fue construido de acuerdo a un diseño elaborado preconcebido y que el sitio sigue un patrón de organización que le otorga una serie de divisiones reconocibles (Fig. 3). La plaza y el ushnu, son el centro de la ciudad, y a partir de ahí se irradia una serie de calles, espacios abiertos y muros. Estas forman esencialmente 'líneas', algunas más claras que las otras, dividiendo al sitio en varias zonas distintas (Morris y Thompson 1985: 72). La 


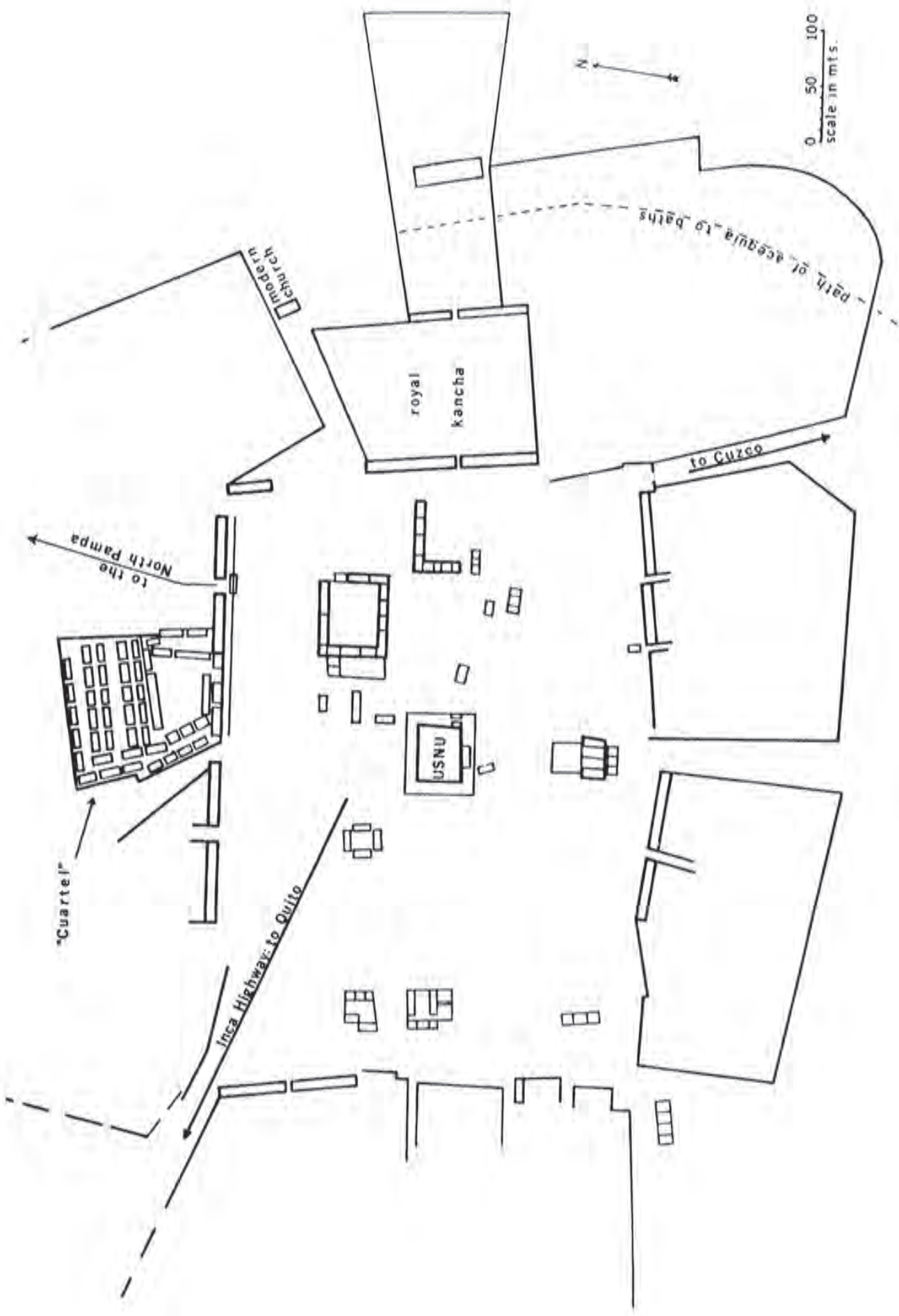

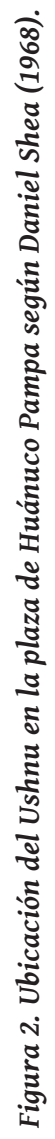


principal división del sitio observada, es la que forma el camino inca principal o Qhapaq Ñan, el cual pasa a través de Huánuco Pampa en su ruta Cusco hacia Quito, el camino, cruza la ciudad diagonalmente en dirección sureste a noroeste con el ushnu al medio.

\section{La Relación del ushnu de Huánuco Pampa con el paisaje}

Las investigaciones previas indican que Huánuco Pampa posee una relación con los equinoccios por tener un eje este-oeste que cruza el sitio e influye en la organización espacial de todo el asentamiento (Harth-Terré 1964; Morris 1987; Morris y Thompson 1970, 1985). Posteriores investigaciones presentan evidencias de alineamientos astronómicos en este sitio, e indicios de principios de organización espacial basadas en orientaciones astronómicas, así como la utilización específica de algunas estructuras como observatorios (Pino 2004b).

El ushnu se encuentra en una posición central en relación a todo el asentamiento inca y como ya se indicó, los ejes principales de orientación de los muros del ushnu están alineados sobre los ejes perpendiculares de las líneas este-oeste y norte-sur con mucha precisión, siendo los acimutes de los mismos: $358^{\circ} 44^{\prime} 12^{\prime \prime}$ al Norte y $88^{\circ} 44^{\prime} 12^{\prime \prime}$ al Este. Es decir con una desviación de $01^{\circ} 15^{\prime} 48^{\prime \prime}$ de los puntos cardinales geográficos, lo cual es una desviación en extremo mínima. Razón por la cual, asumiremos que los ejes de orientación del ushnu son las líneas este-oeste y norte-sur, pues consideramos que esa fue la intención que se tuvo al construir esta plataforma y que se percibe notablemente. El aspecto más destacable del ushnu obviamente es la orientación hacia el Sur de los dos únicos accesos de la plataforma superior y de su única escalinata, por lo que afirmamos que el ushnu está orientado al sur geográfico.

Los accesos y la escalinata además de estar orientados hacia el Sur, se orientan directamente hacia la calle que se dirige al Sur al finalizar la plaza por ese lado, y esa misma orientación se dirige a una pequeña laguna conocida como Piogocha y seguidamente al cerro Colca, donde se encuentran las estructuras de almacenaje. Esa sería una primera apreciación de la orientación al sur del ushnu. Sin embargo, cuando se observa el ushnu y todo el complejo, desde una posición extrema norte del área que circunda a Huánuco Pampa, notamos con claridad, que en la dirección Sur del asentamiento se encuentra el nevado de Yarupajá destacando en la cordillera del Huayhuash, detrás de las colinas al sur del cerro Colca.

Por lo tanto, en base a la ubicación de la cordillera del Huayhuash podría entenderse por qué la orientación principal del ushnu es hacia el Sur. Como se mencionó la parte más importante de esta cordillera es el nevado de Yarupajá, que es el más alto de la zona, con una altitud de $6634 \mathrm{msnm}$, además de ser uno de los nevados más altos de los Andes².

Para afirmar, con más asidero la hipótesis que la intención de orientar el ushnu hacia el Sur es por la posición del nevado Yarupajá, podemos basarnos también en que este nevado además de ser importante por las características ya descritas, fue muy importante a nivel religioso para las poblaciones prehispánicas de la región de Huánuco, según se percibe en las informaciones recogidas en documentos del siglo XVI, para empezar citaremos a Cristóbal de Albornoz (1967 [1582]), quién menciona que fue: «Yarobaha, guaca principal de los indios hurinsayas de Guánoco, es una piedra en un cerro nevado» (Albornoz 1967 [1582]: 30). Contamos también con los testimonios de indígenas del pueblo de San

2 Entre las cumbres más altas de la cordillera de los Andes se encuentran: el Aconcagua con $6962 \mathrm{msnm}$ en Argentina; el Huascarán con 6768 msnm, el Salkantay con 6271 msnm, el Alpamayo con 5947 msnm, el Ausangate con 6372 msnm, el Ampato con 6318 msnm, el Coropuna con 6445 msnm en Perú; el Volcán Llullaillaco con 6739 msnm en Chile; el Chimborazo con 6310 msnm en Ecuador; el Sajama con $6542 \mathrm{msnm}$ en Bolivia. Cabe mencionar que en las proximidades de las cumbres del Aconcagua, Ampato y Llullaillaco se han encontrado ofrendas Incas de sacrificios humanos y demás objetos asociados a rituales de Qhapaq Ucha (Bárcena 2001; Reinhard 2005); y en los alrededores del Coropuna se han encontrado asentamientos Incas que incluían Ushnu (Ziołkowski 2008:122-159). 





Francisco de Otuco de la doctrina de San Pedro de Acas, quienes mencionan que: «todos los susodichos son los que a bisto este testigo hablando con las guacas y a oído deçir a sus pasados que los dichos Guaris primeros eran hombres de nacion gigantes barbados y que su origen fue de Yarupaxa que es vn çerro grande nebado que esta en la cordillera arriba de Mangas» (Noboa 2003: 230 [1656: fol. 13v.]).

Por lo tanto no es de extrañar que la orientación principal del ushnu de Huánuco Pampa fuera hacia el Sur y ex profeso hacia este nevado, al ser este aspecto geográfico no solo huaca principal en la región de Huánuco, sino también una pacarisca o un mítico lugar de origen. Teniendo en cuenta que las pacariscas eran tomadas con mucha consideración por los incas, según lo testimonia Cristóbal de Albornoz quién dice: «el prencipal género de guacas que antes que fuesen subjetos al ynga tenían, que llaman pacariscas, que quieren decir creadoras de sus naturalezas.../ /... Hay entre estas guacas pacariscas muy muchas que reedificaron los ingas, dándoles muchos mitimas servicios que para este fin los mudava de unas provincias a otras. Dioles -el inga- muchos ganados y basos de oro y plata como fue en toda la cordillera que mira al mar, en todo lo que conquisto, en especial a cerros de nieve y volcanes que miran al mar y que salen de los ríos que riegan muchas tierras» (Albornoz 1967 [1582]: 20), por lo que según esta cita Albornoz da a entender, que con ciertas pacariscas, los Incas reapropiaron sus espacios dotándolas de infraestructura y personal para su servicio.

\section{EL USHNU COMO EJE DE PLANIFICACIÓN DE UN CENTRO ADMINISTRATIVO-RELIGIOSO INCA}

En el periodo Inca, el patrón de «establecimiento obligado», fue para Craig Morris, «una característica común muy importante de la organización política y económica andina», característica que nos permite saber que el Estado Inca tuvo mucho poder, que le permitió fundar grandes asentamientos que crecerían rápidamente (Morris 1973: 127). De alguna manera lo que dijo Morris, fue que la estrategia Inca fue exitosa, pero la cuestión que nos interesa saber y definir, es cuales fueron los factores para el éxito. Y para encontrar estos factores, una de las pistas debía estar en la evidencia arqueológica, pues como dijo Morris, las pruebas concluyentes de las informaciones que se encuentra en las crónicas solo se podían encontrar en los datos arqueológicos (Morris 1973: 128).

En estos grandes asentamientos que fundaron los incas, como Huánuco Pampa, sus edificios monumentales y grandes espacios sugirieron a Morris que de algún modo podían ser «ceremoniales» pero que se prefirió denominarlos como «centros urbanos» o «establecimientos urbanos» con un carácter «intrusivo», «obligado» o «impuesto» (Morris 1973: 129), que debían su existencia a un llamado «urbanismo compulsivo» (Makowski 2008: 179), y que eran ocupados temporalmente por grupos de personas que procedían de los pueblos locales circundantes (Morris 1973: 130).

Sin duda estos establecimientos fueron planificados y con una aparición súbita, llamando la atención que en este tipo de establecimientos se logren construir más de mil estructuras, establecimientos que al parecer fueron fundados en serie y en un corto tiempo (Morris 1973: 130), establecimientos que por su planificación se infiere que fueron construidos con una finalidad para «cierto tipo de actividades que no eran fundamentalmente residenciales en el sentido usual» y tampoco se podía observar sectores funerarios (Morris 1973: 132), asentamientos presuntamente urbanos en los cuales de forma limitada el área utilizada con fines «estrictamente habitacionales» no sobrepasó el 10\% del total y el área pública-ceremonial ocupo más del 60\% del área total (Makowski 2008: 178, 180). Establecimientos que poseían un carácter de «artificialidad» por lo que después de la caída del Estado Inca se despoblaron rápidamente, pues el hogar o lugar de origen de los ocupantes temporales se encontraba en otra parte (Morris 1973: 132), por lo que también se ha propuesto que la fundación de este tipo de complejos urbanos planificados parece responder a la decisión política de una autoridad o gobernante, y que al colapso de su mandato, estos asentamientos fueron despoblados (Makowski 2008: 176). 
Para entender los factores del cierto éxito en la rápida construcción de estos establecimientos o asentamientos artificiales y lograr una explicación de sus características particulares, Craig Morris nos plantea dos fórmulas o modos que consisten en: «Uno de ellos es tratar de colocarlos en el contexto de las instituciones Inca en que se desarrollaron y funcionaron. Necesitamos inquirir sus contribuciones esenciales al más amplio sistema económico y político y cómo interactuaban con otras instituciones que condicionaron su forma y determinaron algunas de sus características particulares. El otro modo es investigar más ampliamente para ver si alguna estructura general de desarrollo de las formas de establecimiento y de las instituciones económicas nos sirve» (Morris 1973: 132). Así pues según este autor y buscando entender mejor la estrategia inca, estos establecimientos permitían la centralización de una serie de operaciones administrativas, ceremoniales y económicas sobre la que se sustentaba el Estado, por lo que sitios como Huánuco Pampa eran de importancia capital (Morris 1973: 134). Esta aparente precocidad de las proezas incas, por lo tanto podía ser entendida a partir de las bases institucionales mediante las que se construyeron y poblaron estos centros estatales, que enfatiza su construcción en edificios monumentales ceremoniales y otorga importancia a los espacios de carácter temporal que sirvió para albergar a las personas con las que exitosamente se logró realicen un servicio temporal o mita (Morris 1973: 135), edificios y espacios como plazas cercadas y amplios salones o kallankas destinadas para el uso de visitantes (Makowski 2008: 182). Las actividades en los establecimientos estaban posiblemente vinculadas con un calendario agrícola y ceremonial, actividades en las servían más de 30.000 personas de una forma temporal no residente, con las que el Estado debía ser recíproco y asumir su alojamiento, la comida y bebida (chicha) de estas personas (Morris 1973: 136-137; 1981: 141,146; Morris y Santillana 1978: 76), en un asentamiento con capacidad de entre 9.000 y 15.000 personas (Morris 1981: 148), que era un tipo de lugar en el que se daba albergue y servía como espacio de reunión para élites locales y foráneas en fechas festivas, y cumplir funciones ceremoniales (Makowski 2008: 178-179).

Morris nos deja interrogantes claves para entender este fenómeno del urbanismo obligado, como: ¿Si eran típicos estos asentamientos a lo largo de la red de caminos incaicos?; ¿Cuándo surge en los Andes, este tipo de establecimientos impuestos por el Estado?; o ¿Si este tipo de establecimientos obligados fue una creación inca?, para responder a estos cuestionamientos, el autor nos sugiere que todo esto debe tener sus antecedentes en varios cientos de años (Morris 1973: 137). De la misma forma, la planificación y construcción de estos establecimientos fue supervisada por arquitectos incas y a pesar de ello, hay una variación considerable en las características de cada asentamiento, por lo que nosotros consideramos que para cada región debía haber particularidades en las estrategias de conquista y control; y como también menciona Morris estos establecimientos se construían donde no había una autoridad centralizada y eran ubicadas en lugares que sirvierán de núcleo para el control, y estos conformarán los centros administrativos artificiales «aparentemente urbanos» con una ocupación efímera condicionada a las necesidades y recursos del Estado (Morris 1973: 138-139).

En el caso de Huánuco Pampa, el plano del asentamiento, la existencia de calles y la distribución de ciertos edificios, sugirieron a Craig Morris a pensar que la construcción de este sitio se basó en un modelo o diseño general, en el que algunos sectores parecen haberse ejecutado bajo un rígido control (Morris 1981: 145-146; 1987: 32), pero con la interrogante de por qué la construcción de ciertos edificios fueron construidos en determinadas posiciones y a que se debió su distribución en el espacio (Morris 1987: 27). Según este mismo autor, la función de este sitio fue claramente administrativa, no estando claro cómo se llevó a cabo dicha administración, pero que sin embargo muchas de estas actividades «se hallaban sumergidas en un elaborado ceremonial, derivado en gran parte de tradiciones andinas que posiblemente ya eran muy antiguas en la época Inca» (Morris 1981: 148-149). Las formas de las vasijas procedente de las excavaciones en el sector este, ofrecieron datos para afirmar que las actividades preponderantes fueron la preparación de comida y chicha en escalas masivas, que indicarían una determinada importancia en la organización de fiestas y ceremonias públicas como base para la articulación de las relaciones sociales y políticas, por lo que posiblemente estas costumbres y principios de organización se vean reflejadas a niveles urbanos y estatales (Morris 1981: 149). Por lo que un aspecto 
fundamental para que funcionara este tipo de administración era corresponder con una «elaborada hospitalidad estatal» a los que llegaban a Huánuco Pampa para los diversos fines y proporcionarles la suficiente comida y bebida (Morris 1981: 150). De todos los productos suministrados, la chicha fue el más importante, pues este constituyó el «elemento esencial en un centro donde el trabajo del Estado se llevaba a cabo a menudo dentro de un contexto ceremonial», y que su producción ha sido evidenciada en diferentes sectores del asentamiento, como una de las actividades de mayor importancia (Morris y Santillana 1978: 76-77; Morris 1987: 31).

Un aspecto que es evidente, y que llama la atención sobre Huánuco Pampa, es que todo el asentamiento está construido alrededor de su inmensa plaza $(520$ × $360 \mathrm{~m})$ con la plataforma ushnu al centro de la misma, a partir del cual, de forma radial, están organizados los diferentes sectores divididos por espacios abiertos a manera de calles (Morris 1987: 33). Evidentemente las actividades realizadas en el sector central y en los sectores que la rodean son los indicadores para entender esta organización del espacio y las funciones específicas. Las indicaciones de la información arqueológica sugieren que esta organización del espacio tuvo que ver con la religión y específicamente ajustar el asentamiento a un eje solar, equinoccial, y que esta organización espacial además de ser el reflejo de una cosmología particular haya servido de forma simbólica para la manipulación de las poblaciones que acudían al sitio a brindar servicios (Morris 1987: 42-43), servicios y bienes que generaban el desplazamiento anual de grupos de población regulados por «nutridos calendarios ceremoniales» y «eficientes» ideologías religiosas (Makowski 2008: 179).

Por lo mencionado entonces, el carácter más idóneo con el cual designar a Huánuco Pampa y de la forma más completa sería el de llamarlo «centro administrativo-religioso», por las características descritas y tal como algunos investigadores categorizan este tipo de sitios, que se ajustan a funciones de un centro ceremonial y capital a la vez (Makowski 2000: 109; 2008: 178-179). Por lo que Huánuco Pampa puede ser categorizado como un centro ceremonial, que con mucha probabilidad, al fundarse haya transformado y convertido el paisaje local en un «escenario sagrado» en el que los servicios que se entregaron, posiblemente adquirieron el carácter de una obligación religiosa, en el que como escenario sirvió para transmitir una determinada ideología religiosa e inscribir en el paisaje una memoria social particular que sirvió como instrumento de poder (Makowski 2008: 179). Vale la pena enfatizar que en este sitio, la presencia de una plaza de tamaño notable, grandes patios y plataformas escalonadas (que además del ushnu se encuentran cuatro más rodeando inmediatamente el sitio), son formas arquitectónicas que con mayor recurrencia están presentes en sitios ceremoniales, tal como lo ha sido percibido Makowski (2008: 180). Por lo que entender el carácter de ceremonialidad de este sitio serviría también para entender su particular y ficticia apariencia urbana, y proponer más bien que los grupos de poblaciones que acudían a sitios como Huánuco Pampa, asistían periódicamente «para cumplir sus obligaciones en un ambiente festivo y/o rendir honores a los ancestros» (Makowski 2008: 181).

Para entender el éxito súbito del Estado inca en ciertas partes de la región del Chinchaysuyu, tentativamente podemos decir que Huánuco Pampa fue un centro ceremonial que por alguna razón rápidamente adquirió prestigio, que sirvió como lugar de reunión de varias poblaciones que asistían a banquetes, que con mucha probabilidad los momentos en que fue construido fueron fechas festivas, que en este escenario a través de trabajos comunales se plasmó una relación directa entre la arquitectura y el paisaje, y se llevó a cabo cultos importantes con fines estatales; afirmaciones que se basan en reflexiones sobre ciertos centros ceremoniales en los Andes con apariencia urbana (Makowski 2008: 182), en el que los espacios públicos jugaron un rol importante en las relaciones sociales entre el Estado y las poblaciones locales, y estos espacios públicos están físicamente al centro del asentamiento y obviamente están reflejando una intención protagónica para estos espacios, intención que se percibe fue concebida aun desde el diseño o modelo con el cual fue creado.

Podemos plantear entonces que, de alguna manera se quería mostrar y enfatizar la importancia de la centralidad del espacio público y construir sobre el mismo otro escenario aún más evidente 
en el que se lleven a cabo las acciones más importantes a nivel público y por lo tanto sean visibles, efectivas y no cuestionables. En base a lo mencionado líneas arriba, podemos decir que la función del espacio central del asentamiento de Huánuco Pampa fue eminentemente ceremonial, en el que, con todas las evidencias mencionadas, se realizaron prácticas rituales. Entendiéndose el ritual como una práctica social conformada por un «conjunto de actos y expresiones formalizados, estandarizados, repetitivos y ordenados en forma secuencial por cuyo medio se trasmite y comunica información significativa entre sus participantes», y que este haya servido como un vehículo poderoso para congregar y movilizar entidades colectivas, convirtiéndose también de alguna manera el ritual en un escenario con poder persuasivo, escenario en el que se lleva a cabo performances que resaltaban y comunicaban una autoridad social (Vega-Centeno 2008: 93), y estos escenarios a través de los rituales se convertían de igual manera en un espacio común de convergencia.

Huánuco Pampa sirvió como un escenario de festines, compromisos y alianzas, en un sitio diseñado de acuerdo a cánones ceremoniales más que «urbanos» (Pino 2010b). Las actividades festivas fueron sin duda las actividades en el que se desarrollaban los diferentes rituales necesarios para conseguir la participación de los grupos de población en los trabajos requeridos. Así como posiblemente las fiestas fueron el vehículo con el que rápidamente se consiguió el prestigio necesario para lograr un liderazgo y emprender la construcción de un asentamiento preconcebido o planificado como quiera llamársele. Con las fiestas organizadas a gran escala, muy probablemente se consiguió comprometer a los líderes locales que asistían y que eran agasajados, repitiéndose estas fiestas de forma periódica y constante para así lograr el la construcción o crecimiento rápido del asentamiento.

En un contexto festivo y de relaciones sociales, cabe resaltar aún más el rol del brindis y la bebida o de la libación en el sentido ritual, pues los rituales de libación constituían el acto principal para abrir y cerrar las negociaciones en los Andes, sobre todo en las que participaban los curacas locales con las autoridades incas (Cummins 2004: 166). Aun en fechas recientes, la información etnográfica de hace unas tres décadas atrás, indica que en las alturas de Ayacucho, en Chuschi «la ebriedad es un deber ritual de los «envarados» o autoridades con cargos temporales, que debían de preparar y proveer abundante chicha en los trabajos y en las fiestas que les tocaban ejecutar y beber con toda la población como un deber obligatorio (Isbell 1971: 287-293). En los Andes prehispánicos los que asistieron a las actividades festivas, posiblemente bebieron exageradas cantidades de chicha, tan es así que el beber en demasía incluso causó asombró para los españoles recién llegados, que las calificaban de grandes borracheras, que constituían para ellos como eventos de libación indiscriminada (Cummins 2004: 70; Segura 2001: 159).

Las evidencias etnohistóricas y arqueológicas nos indican que existió espacios para estos fines de libación en contextos festivos y ceremoniales, por lo que el espacio de libación más importante debía, a todas luces, estar en una posición central y ser el primer lugar en la planificación y construcción. Al ser esto cierto, consecuentemente, la evidencia estaría indicando que el brindis o la libación era lo más importante en el diseño y función de este tipo de asentamientos. En Huánuco Pampa, por lo tanto, el beber ritualmente era la institución clave para el desarrollo de cualquier acción mancomunada y de gran envergadura; por lo mismo, el espacio de libación, que serviría para articular los poderes locales (poderes de huacas o líderes), debía ser público y tener un carácter de centralidad.

Según lo presentado en este artículo, en Huánuco Pampa, en el centro del asentamiento estuvo construido el ushnu, que por las características de la ubicación, su articulación con los demás sectores del asentamiento y su relación con el paisaje con sus montañas principales, esta construcción fue posiblemente la primera en ubicarse y construirse. (Fig. 4).

Las evidencias etnohistóricas (Pino 2010) apuntan a que, por la multifuncionalidad del ushnu, esta construcción a nivel público fue la más importante, por lo tanto, tenía cierto carácter de centralidad tal como lo propuso Hyslop (1990: 69-101). Sin embargo, cabe recalcar que la función principal del ushnu fue de servir como escenario o espacio para los rituales de libación más importantes, sobre todo de las deidades consideradas ancestros (ya sean astros, ídolos, bultos, o momias) y libaciones a nivel 


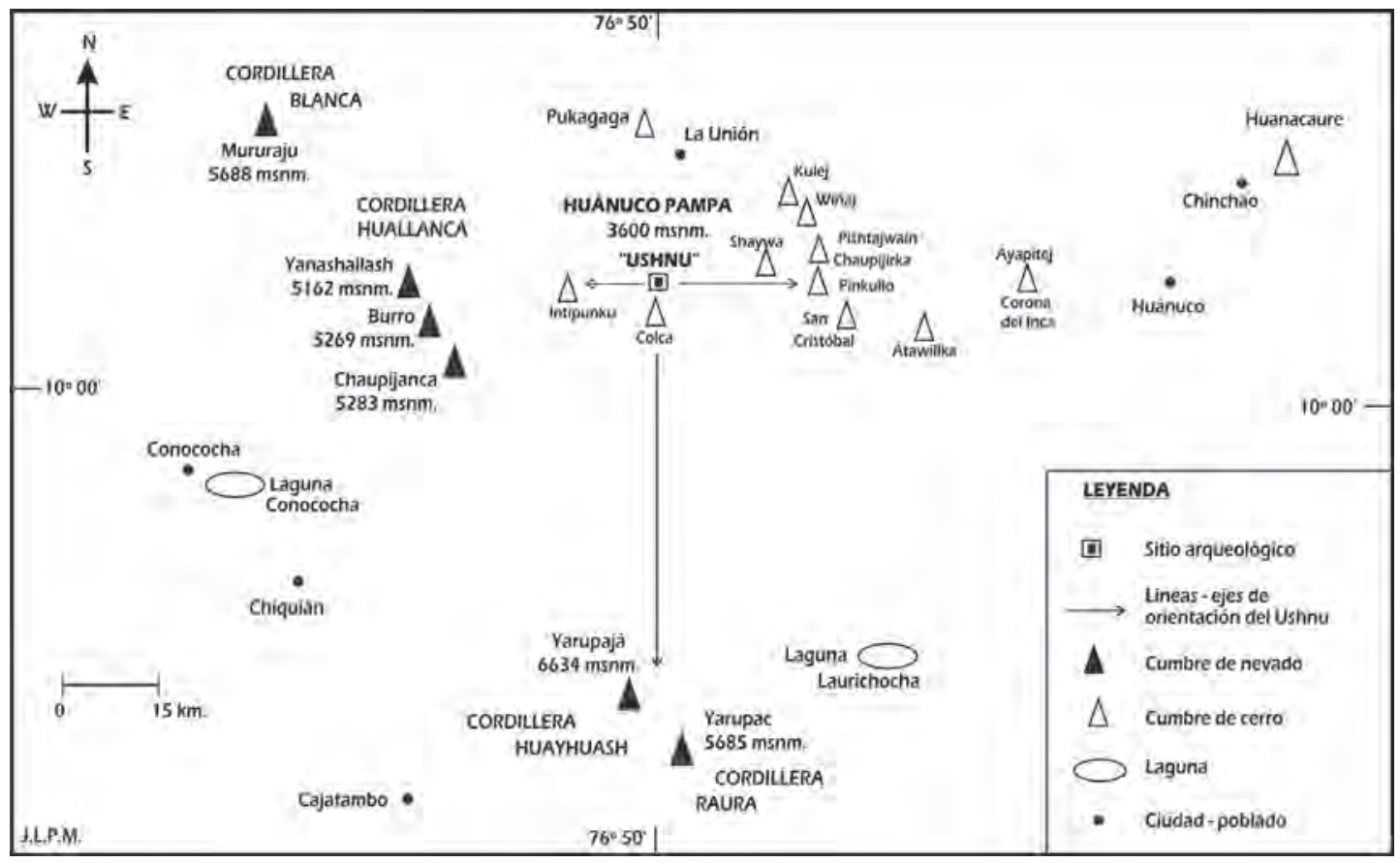

Figura 4. Ubicación del Ushnu de Huánuco Pampa en relación al paisaje local.

público de mucha importancia (Cummins 2004: 157). Además, su diseño complejo, en algunos casos tuvo propósitos e instalaciones particulares para la recepción y drenaje de ofrendas líquidas, tal como se observa para el caso de Huánuco Pampa.

Ciertas preguntas son necesarias formular en el caso de Huánuco Pampa, como: ¿Por qué el ush$n u$ tiene un espacio plano tan grande $(48 \times 32 \mathrm{~m})$ en su plataforma superior, a manera de un cercado o kancha, y dos accesos amplios?, es que acaso los personajes que accedían a esta construcción eran en mayor número que en otras plataformas de lugares similares. ¿Tendría algo que ver el enorme tamaño de la construcción (6144 $\mathrm{m}^{3}$ aproximadamente) con el prestigio del sitio y de las poblaciones locales?. Los personajes, ídolos y momias que accedían al ushnu y brindaban con chicha, servían de mediadores en las negociaciones realizadas. Definitivamente el ushnu, fue el espacio para los brindis más importantes (entre mortales y deidades, entre líderes principales y ancestros), y por su ubicación en Huánuco Pampa, proponemos que esta construcción (de por sí compleja y multifuncional según las evidencias para la época Inca) fue el principio de planificación de este tipo de centro administrativo-religioso en el Chinchaysuyu al ser el centro principal de los brindis y fiestas. La chicha, así como el beber y brindar en ceremonias, sin duda fue de importancia capital en las relaciones sociopolíticas en los Andes (Cummins 2004: 69-72, 152), sobre todo es lo que se infirió y se afirma partir de las evidencias arqueológicas de muchos sitios (Segura 2001; Jennings y Browser 2008: 7), y que la libación de chicha fue parte de una efectiva estrategia económica centrada en la hospitalidad, sobre todo para el emprendimiento de grandes obras públicas de una manera rápida y eficaz (Segura 2001: 174).

El ushnu en Huánuco Pampa ocupó un espacio físico central en medio de una plaza que habría sido el escenario «donde los diferentes grupos podían representar relaciones de complementariedad y de oposición, mediante danzas, procesiones y batallas rituales» (Morris y Covey 2003: 133). Sin embargo, cabe recalcar a su vez que el ushnu fue el lugar principal de posicionamiento del Sapa Inca, personaje con carácter 
divino y que era la autoridad máxima, que según varios investigadores (Martínez 1995), estos concuerdan que este estaba íntimamente relacionado a la idea de «orden» y recalcado como opuesto a la idea de «desorden», tal como indicó Jan Szeminski (1992, 1993), que Inca poseía dos significados principales, como el de ser hijo del Inti (deidad, que es el Sol en muchos casos) que gobierna o manda y que es asimismo el principio ordenador del mundo. A su vez según los estudios de Franklin Pease, Inca era el representante del Inti en la Tierra, considerado como un arquetipo que modelaba la conducta de los hombres, con una simbología asociada a la construcción y capaz de transformar la faz de la tierra o el paisaje (Pease 1978: 69-70). Asimismo, si el ushnu fue el lugar posición de las autoridades, y que esta estructura estaba dedicada para las libaciones al Sol y era uno de los lugares donde también se colocaba a su ídolo, entonces se puede entender que el Sol adoptaba la imagen de autoridad y que estaba directamente bebiendo a través del ushnu con todos los líderes locales que asistían a las festividades, acción de libación facilitada por el diseño complejo de la plataforma-ushnu con un receptáculo de cantos rodados y sus ductos de drenaje correspondiente. Por lo tanto las propuestas presentadas apoyan el argumento de la estratégica ubicación del ushnu en Huánuco Pampa con una estrecha relación al paisaje circundante y el correspondiente propósito y diseño de estos asentamientos más con un carácter religioso y ceremonial.

\section{A MANERA DE CONCLUSIONES}

Por el carácter ceremonial, el ushnu de Huánuco Pampa sirvió no solo de centro de las actividades públicas que se realizaron durante el año, sino que inicialmente el espacio correspondiente al ushnu sirvió de centro del asentamiento y su centro exacto sirvió para la entera planificación del sitio. La plataforma que constituía el ushnu, estuvo vinculada con los aspectos más notables del paisaje circundante a través de las líneas que se proyectaban mediante los rasgos más notorios de sus elementos arquitectónicos y principales direcciones del asentamiento. La dirección Sur de su escalinata posiblemente estuvo orientada al nevado Yarupajá, que fue una huaca pacarisca local muy importante.

Las actividades públicas que se realizaron en torno al ushnu de Huánuco Pampa, posiblemente constituyeron una forma simbólica de retribuir a las poblaciones presentes, en el que los rituales de libación entre los curacas principales con el Estado Inca y sus deidades, fueron parte esencial de una reciprocidad asimétrica mediante la cual se logró las alianzas con las poblaciones locales para los fines estatales. Los rituales de libación y festividades constituyeron una parte importante de las relaciones Incas. El ushnu en Huánuco Pampa, muy probablemente, fue el centro principal de las festividades y rituales de libación, por lo tanto debió constituirse como un principio importante de planificación de un centro administrativo-religioso Inca en el Chinchaysuyu.

\section{Agradecimientos}

Quiero agradecer a los profesores Julian Idilio Santillana, Krzysztof Makowski y Marco Curatola del Programa de Estudios Andinos por el asesoramiento en la investigación. A Hernán Ramos, Carlo Ordoñez, Wendy Moreano, Gabriel Ramón y Martha Bell, por el apoyo en campo, los pertinentes comentarios en el presente estudio y las múltiples facilidades con el material bibliográfico. Y un agradecimiento especial a Ramiro y Norma Matos por su apoyo constante. 


\section{BiBLIOGRAFÍA}

ALBORNOZ, Cristóbal

1967 [1582] «La Instrucción para descubrir todas las guacas del Pirú y sus camayos y haziendas», editado por P. Duviols. Journal de la Société des Américanistes 56: 9-39.

CUMMINS, Thomas B. F.

2004 Brindis con el Inca. La abstracción andina y las imágenes coloniales de los queros. Lima: UNMSM.

GASPARINI, Graziano y Luise MARGOLIES

1977 Arquitectura Inca. Caracas: Centro de Investigaciones Históricas y Estéticas, Facultad de Arquitectura y Urbanismo. Universidad Central de Venezuela.

HARTH TERRE, Emilio

1964 «El pueblo de Huánuco Viejo». En: El Arquitecto Peruano N³20/21: 1-20. Lima.

HYSLOP, John

1990 Inca settlement planning. Austin: University of Texas Press.

ISBELL, Billie Jean

1971 «No servimos más...». En: Revista del Museo Nacional Tomo 37. Lima: INC.

JENNINGS, Justin y Brenda BROWSER

2008 «Drink, Power, and Society in the Andes: An Introduction». En: Justin Jennings y Brenda Browser (eds.) Drink, Power, and Society in the Andes. University Press of Florida.

MATOS, Ramiro

1986 «El ushnu de Pumpu». Cuicuilco 18: 45-61.

1994 Pumpu, Centro Administrativo de la Puna de Junín. Lima: Editorial Horizonte, BCR y Taraxacum.

1995 «Los Inka de la Sierra Central del Perú». Revista de Arqueología Americana 8: 159-190.

MATOS, Ramiro, Carmen ARELLANO y David BROWN

1996 «Asentamientos inka en Chakamarka y Tarmatambo (departamento de Junín). Problemas y criterios de interpretación para la reconstrucción de una provincia Inka». I Encuentro Internacional de Peruanistas Tomo I: 181-193. Lima: Universidad de Lima, FCE.

MAKOWSKI, Krzysztof

2000 «El síndrome de Çatal Hüyük: Observaciones sobre las tendencias aglomerativas tempranas». En Arqueología y Sociedad 13: 99-108. Lima: Museo de Arqueología y Antropología, UNMSM.

2008 «La arquitectura pública del periodo precerámico tardío y el reto conceptual del urbanismo andino». En: P. Kaulicke y T.D. Dillehay (eds.), Procesos y expresiones de poder, identidad y orden tempranos en Sudamérica, Boletín de Arqueología PUCP N 10 (2006): 167-199, Lima.

MAKOWSKI, Krzysztof y Clive RUGGLES

2011 «Watching the sky from the ushnu: the sukanka-like summit temple in Pueblo Viejo-Pucara (Lurin Valley, Peru)». En: C.L.N. Ruggles (ed.) Archaeoastronomy and Ethnoastronomy : Building Bridges between Cultures: Proceedings IAU Symposium No. 278, Oxford IX International Symposium on Archaeoastronomy.

MAKOWSKI, Krzysztof, Maria Fe CÓRDOVA, Patricia HABETLER y Manuel LIZARRAGA

2008 «La plaza y la fiesta: reflexiones acerca de la función de los patios en la arquitectura pública prehispánica de los periodos tardíos». En: P. Kaulicke y T.D. Dillehay (eds.), Encuentros: Identidad, poder y manejo de espacios públicos, Boletín de Arqueología PUCP Nº 9 (2005): 297-333, Lima.

MARTÍNEZ, José Luis

1995 Autoridades en los Andes, los atributos del Señor. Lima: PUCP.

MEDDENS, Frank

1997 «Function and meaning of the usnu in Late Horizon Peru2. Tawantinsuyu 3: 5-14.

MEDDENS, Frank, Nicholas BRANCH, Cirilo VIVANCO, Naomi RIDDIFORD y Rob KEMP

2008 «High Altitude Ushnu platforms in the departament of Ayacucho Peru, structure, ancestors and animating essence». En: J. Staller (ed.) Pre-Columbian Landscapes of Creation and Origin, pp. 315-355. Editorial Springer. 


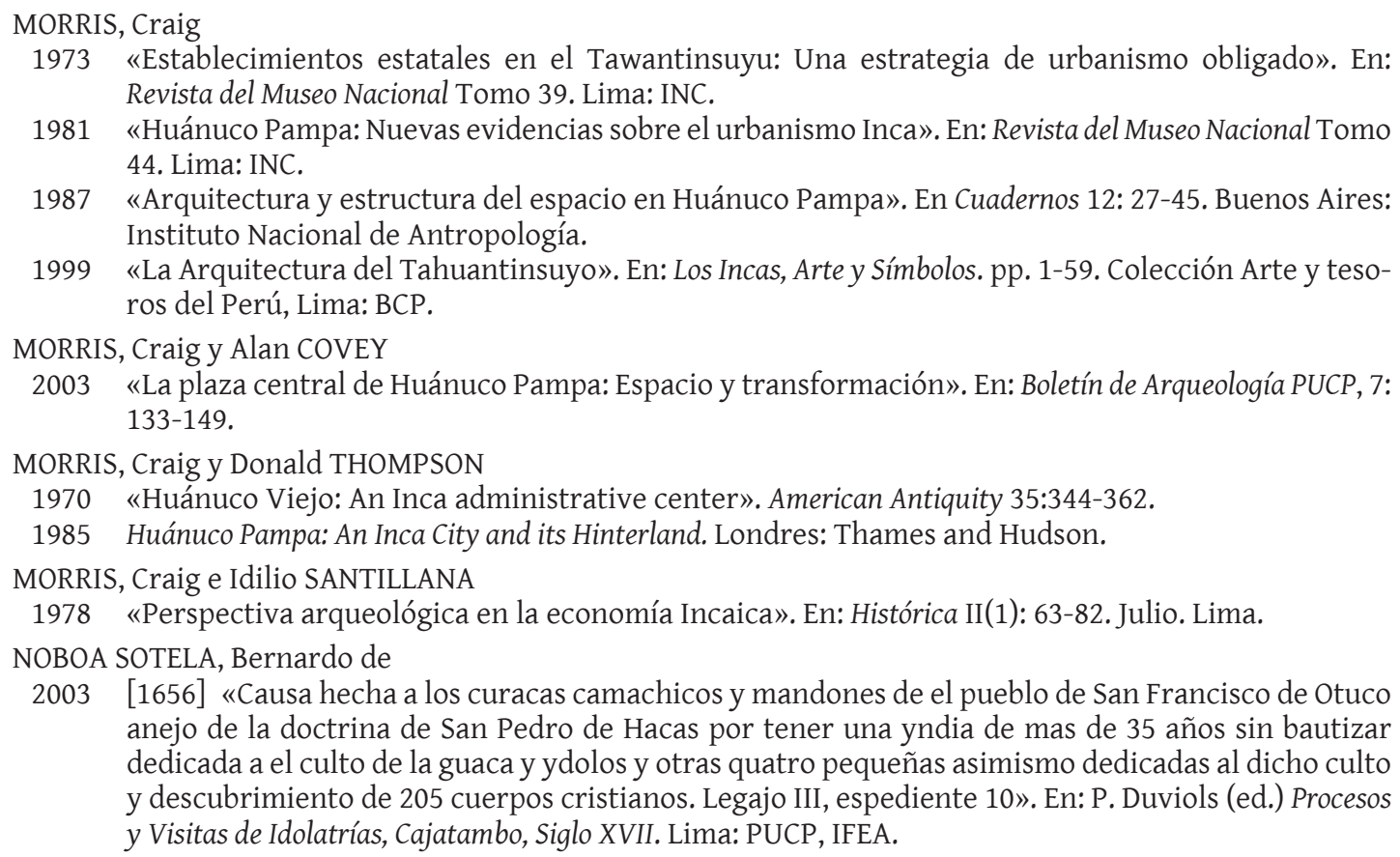

PEASE G.Y., Franklin

1978 Del Tawantinsuyu a la Historia del Perú. Lima: IEP.

PINO MATOS, José Luis

2004a «El Ushnu Inca y la organización del espacio en los principales Tampus de los Wamanis de la sierra central del Chinchaysuyu». En: Chungara Revista de Antropología Chilena, 36(2): 303-311. Arica: Universidad de Tarapacá.

2004 b «Observatorios y Alineamientos Astronómicos en el Tampu Inca de Huanuco Pampa, Perú». En: Revista Arqueología y Sociedad 15: 173 -190. Lima: Museo de Arqueología y Antropología, UNMSM.

2005 «El Ushnu y la organización espacial astronómica en la sierra central del Chinchaysuyu». En: Estudios Atacameños 29: 43-161. San Pedro de Atacama: Universidad Católica del Norte.

2010a «Yllapa Usno: Rituales de libación, culto a ancestros y la idea del Ushnu en los Andes según los documentos coloniales de los siglos XVI-XVII». En: Revista Arqueología y Sociedad 21. Lima: Museo de Arqueología y Antropología, UNMSM.

2010 b «uánuco Pampa: Un escenario diseñado para fiestas y brindis, como estrategia de articulación socio-política en el Chinchaysuyu». En: Revista Inka Llaqta 1(1). Lima.

RAFFINO, Rodolfo, D. GOBBO, R. VAZQUEZ, A. CAPPARELLI, V. MONTES, R. ITURRIZA, C. DESCHAMPS y M. MANNASERO

1997 «El Ushnu de El Shincal de Quimivil». Tawantinsuyu Vol 3: 22-39.

ROWE, John

1990 «Los monumentos perdidos de la plaza mayor del Cuzco incaico». En: Saqsaywaman 3: 81-109. Cusco.

2003 «Hawkaypata: Como fue la plaza de los Incas». En: Los Incas del Cuzco, Siglos XVI-XVIII: 231-235. Cusco: INC.

SEGURA, Rafael

2001 Rito y Economía en Cajamarquilla. Investigaciones arqueológicas en el conjunto arquitectónico Julio C. Tello. Lima: Fondo Editorial PUCP.

SZEMINSKI, Jan

1992 «La transformación de los significados en los Andes centrales (siglos XVI-XVII)». En: Gutierrez Estevez y León Portilla (eds.) De Palabra y Obra en el Nuevo Mundo. Madrid: Editorial Siglo XXI.

1993 La Utopía Tupamarista. Lima: PUCP. 
SHEA, Daniel

1966 «El Conjunto Arquitectónico Central en la Plaza de Huánuco Viejo». Cuadernos de Investigación 1: 108-116.

1968 The Plaza Complex of Huanuco Viejo. Thesis de Master of Science. University of Wisconsin. USA.

VEGA- CENTENO, Rafael

2008 «Consumo y Ritual en la construcción de espacios públicos para el periodo arcaico tardío: El Caso de Cerro Lampay». En: P. Kaulicke y T.D. Dillehay (eds.), Encuentros: Identidad, poder y manejo de espacios públicos, Boletín de Arqueología PUCP Nº 9 (2005): 91-121, Lima.

VILLACORTA, Luis Felipe

2005 «Palacios y ushnus: curacas del Rímac y gobierno inca en la costa central». En: P. Kaulicke, G. Urton I. Farrington (eds.), Identidad y transformación en el Tawantinsuyu y en los Andes Coloniales. Perspectivas arqueológicas y etnohistóricas. Segunda parte, Boletín de Arqueología PUCP N 7 (2003): 151-187. Lima.

ZIOŁKOWSKI, Mariusz

2008 «Coropuna y Solimana: los oráculos de Condesuyos». En: Marco Curatola y Mariusz Ziołkowski (eds.) Adivinación y oráculos en el mundo andino antiguo. pp. 122-159. Lima: PUCP -IFEA.

ZUIDEMA, Tom

1980 «El Ushnu». Revista de la Universidad Complutense 28 (117): 317-362. Madrid. 